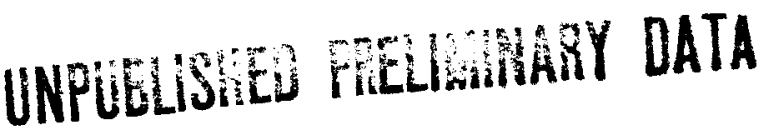

ON THE ORIGIN OF THE CANYON DIABLO NO. 2 AND NO. 3 METEORITES

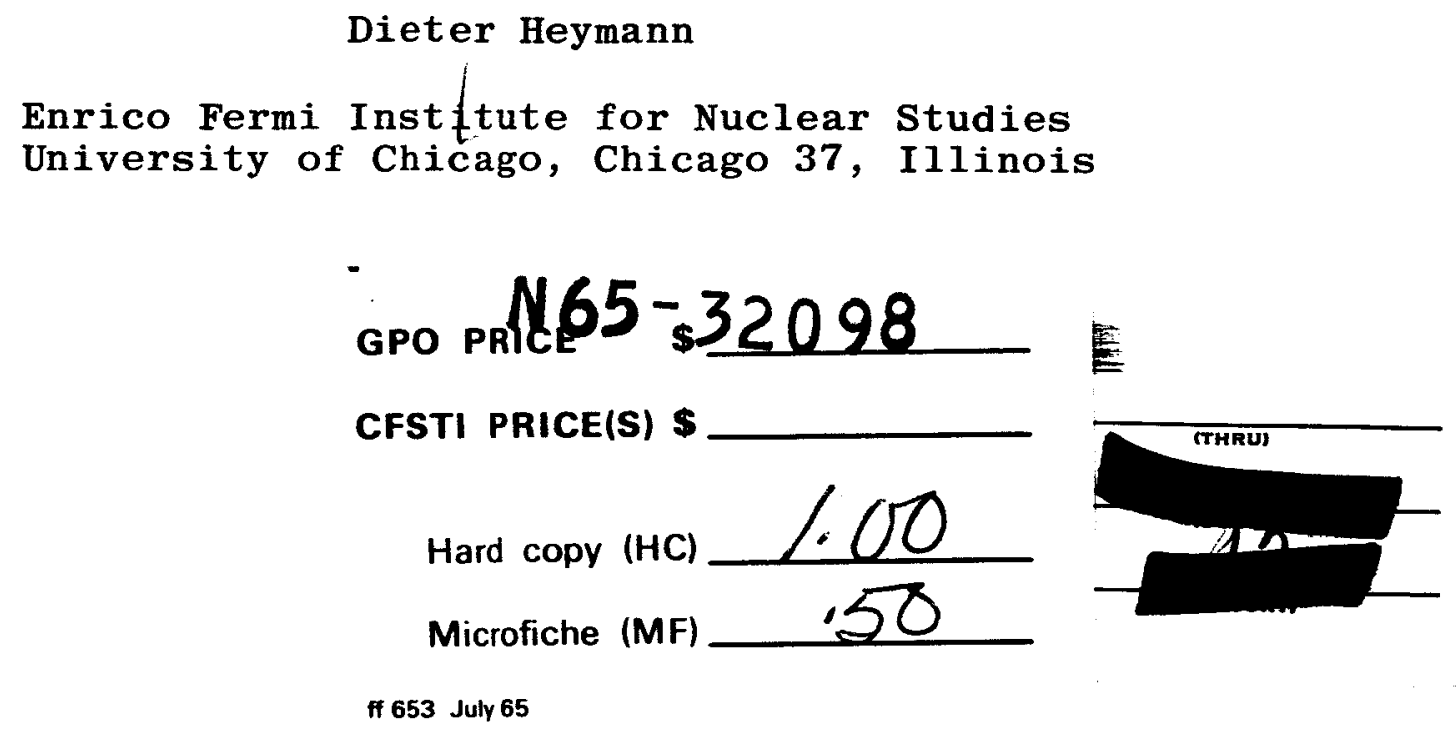

Submitted to Science

July 1964

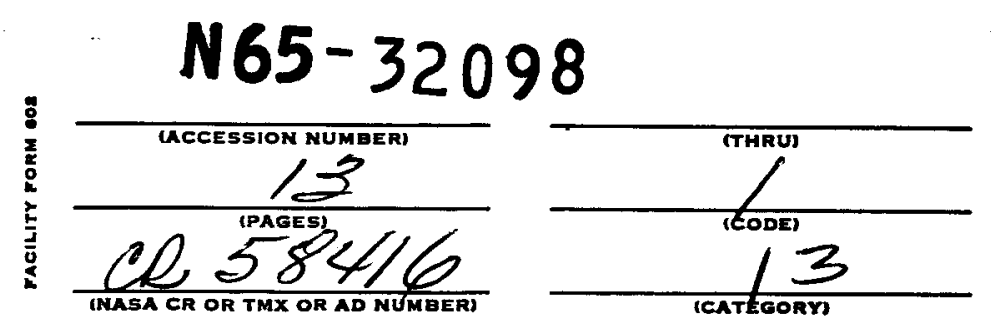

AEC Contract AT(11-1) 382

NASA Grant NsG-366 Research

EFINS $64-38$
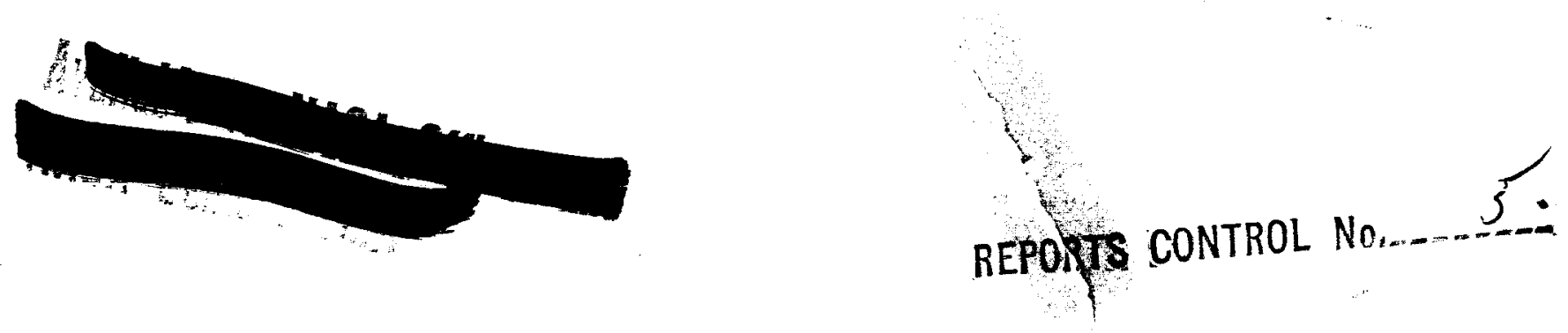
On the Origin of the Canyon Diablo No. 2 and No. 3

Meteorites

Dieter Heymann

32098

Abstract. Measurements of $\mathrm{He}^{3}, \mathrm{Ne}^{21}$ and $\mathrm{Ar}^{38}$ in nine Canyon Diablo meteorites indicate that the "anomalous" Canyon Diablo No. 2 was located inside the main mass of Canyon Diablo "No. 1" when the latter struck the atmosphere. Canyon Diablo No. 3 probably originated from the main mass too, but the evidence is less certain. These conclusions speak against the view that the anomalous Canyon Diablo irons were either satellites of the main mass or distinct falls. It appears instead that significant chemical and structural variations existed in the nickel-iron phase of the meteorite parent body over distances of some tens of meters.

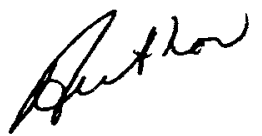

Of the thousands of irons that have been recovered from the Arizona Meteor Crater the vast majority are coarse octahedrites. However, careful searches by Nininger have turned up a small number of anomalous individuals designated as Canyon Diablo No. 2 and No. $3(1,2)$. Both are medium octahedrites; they have a higher nickel content than Canyon Diablo No. 1 and often show a distorted and granulated structure.

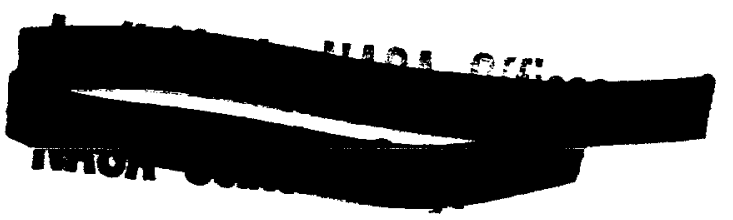


There is no doubt that Canyon Diablo 2 and 3 are truly distinct from Canyon Diablo 1. The kamacite bands of Canyon Diablo 1 vary in width, ranging up to as much as 4 or $5 \mathrm{~mm}$; whereas the kamacite bands in Canyon Diablo 2 average $1.4 \mathrm{~mm}$ in width. The nickel content of Canyon Diablo 1 is $7.11 \%$ as against $8.09 \%$ in Canyon Diablo 2 , and the palladium concentrations are $3.98 \mathrm{ppm}$ and $5.30 \mathrm{ppm}$ respectively (3). Nininger explained these chemical and structural differences in two ways. He suggested that Canyon Diablo 2 was a swarm of small iron meteorites, captured by the main mass while the latter was in orbit around the sun. He also offered the alternative explanation that Canyon Diablo 2 and 3 were distinct falls but thought this possibility less likely. The capture hypothesis was not widely accepted because of the obvious unlikelihood of such an event. Goldberg et al pointed out that the terrain around Meteor Crater is possibly the most extensively searched area for meteoritic material. This area is about 65 square kilometers or $1.3 \times 10^{-7}$ times the earth's surface. A fall frequency on the earth of about 35 iron meteorites per year $(4,5)$ means that this area of 65 square kilometers was hit on the average once in 230,000 years and twice in 460,000 years. From other studies (6) it appears possible that iron meteorites can survive on the surface of the earth for a million years or longer. The hypothesis of a distinct fall is therefore not altogether unlikely.

The evidence evidence for distinct origins of Canyon Diablo 1,2 and 3 is not very convincing, though, despite the chemical 
and structural differences. Canyon Diablo is one of the largest iron meteorites known. Pieces of the parent mass may have been spalled off during passage through the atmosphere and fallen near Meteor Crater. There is considerable disagreement as to the size of the projectile that produced the crater. Öpik (7) estimates a mass of 2.6 million tons, Shoemaker (8) gives 63,000 tons and Bjork (9) places the mass between 30,000 and 194,000 tons, depending on impact velocity. The lowest estimate corresponds to a diameter of 19.5 meters. Thus it appears almost certain that the diameter of the projectile was at least ten meters. The three Canyon Diablo varieties might have been broken out from diametrically opposed locations and since nothing is really known about chemical and structural variations of iron meteorites on such a scale, a third hypothesis deserves consideration in my opinion, namely the one originally rejected: that Canyon Diablo 1,2 and 3 did all come from the same object.

An essential difference among these hypotheses is that the first two assume the anomalous Canyon Diablo to have existed in space as small individual entities, whereas the third one assumes them to have resided in a single, large parent mass. These two possibilities can be distinguished experimentally inasmuch as they should result in markedly different concentrations of cosmogenic rare gases. Signer and Nier (10) have calculated the distribution of the cosmogenic nuclides $\mathrm{He}^{3}, \mathrm{Ne}^{21}$ and $\mathrm{Ar}^{38}$ in spherical meteoroids of different diameters and their results are shown in Fig. 1 . 

lie close to a Signer-Nier curve of infinite mass. Note that each sphere of given mass (masses range from $100 \mathrm{~kg}$ to infinity, i.e. $\gg 2 \times 10^{5} \mathrm{~kg}$ ) has a maximum $\mathrm{He}^{3} / \mathrm{Ne}^{21}$ ratio which occurs at the center of the sphere.

In Fig. I I have also plotted my experimental results on seven different Canyon Diablo 1 specimens. A detailed discussion of the experimental procedure will appear elsewhere (12). Evidently the results agree quite well with a signer-Nier curve for a meteoroid of very large mass. The only specimen which is definitely outside the correlation is 34.4341 . From the mean displacement of the Canyon Diablo 1 points to the left of the Signer-Nier curve for infinite mass, I conclude that the normal Canyon Diablo has a lower exposure age than Grant, namely $540 \pm 100$ million years, which is not in disagreement with a recently measured exposure age of $665 \pm 65$ million years by the $K^{40}-K^{41}$ method (13). Also shown in Fig. 1 are results obtained on samples of Canyon Diablo 2 and 3 . The results of individual measurements are given in Table 1 .

The recovered mass of Canyon Diablo 2 is a few kilograms. I take this to mean that the meteorite was small, less than 500 kilograms if it was a "satellite" or a distinct fall, unless most of its mass disappeared through weathering. The $\mathrm{He}^{3}$ content of Canyon Diablo 2, specimen 371.2 , is about 8 times lower than the highest $\mathrm{He}^{3}$ concentration measured in a Canyon Diablo iron: $301 \times 10^{-8} \mathrm{cc}$ STP/g (34.4341). This result, taken alone, does not necessarily place Canyon Diablo 2 inside the main mass, 
since it is conceivable that this meteorite is a distinct fall and has a cosmic-ray exposure age of only $64 \pm 12$ million years. But this is unlikely. For one thing, all exposure ages of medium octahedrites measured thus far are uniformly higher than 300 million years (14). Moreover, the $\mathrm{He}^{3} / \mathrm{Ne}^{21}$ ratio of this specimen, $108 \pm 7$, is much higher than what one would expect to find inside a meteorite of a few hundred kilograms. The highest ratio observed in Grant (recovered mass 480 kilograms) is $82 \pm 4$ (10). Thus the $\mathrm{He}^{3} / \mathrm{Ne}^{21}$ ratio makes it extremely likely that specimen 371. 2 was once inside a large mass: $10^{4}$ kilograms or larger. Finally, the Canyon Diablo 2 point lies remarkably close to the curve defined by Canyon Diablo 1 . In view of these facts, I conclude that the simplest explanation for the observed cosmogenic rare gas abundances is that Canyon Diablo 2 was once part of Canyon Diablo 1; was located approximately $50 \mathrm{~cm}$ below its preatmospheric surface; and was exposed to cosmic-ray irradiation for the same length of time as the main mass.

In the case of Canyon Diablo 3 , the conclusion cannot be as definite. This iron has the third highest cosmogenic rare gas abundance of 47 Canyon Diablo individuals which I have examined. Moreover, its $\mathrm{He}^{3} / \mathrm{Ne}^{21}$ ratio does not fit well with the curve defined by Canyon Diablo 1 , as can be seen from Fig. 1 . The $\mathrm{He}^{3} / \mathrm{Ne}^{21}$ ratio of 92 is higher than what one would expect for this specimen: 82. Yet, one detail in Fig. 1 leads me to the tentative conclusion that Canyon Diablo 3 also belonged to 
the main mass. Specimen 34.4341 seems to be displaced to the right of the Canyon Diablo 1 curve, yet it appears to belong to the normal variety. This could be explained in two ways. Either Canyon Diablo was broken out from its parent body 1.0 aeon ago and suffered a second collision 540 million years ago (15) or specimen 34.4341 was located in a protrusion on the surface of the main mass. It can be easily shown that in the latter case the production ratios of cosmogenic nuclides are determined by the size of the protrusion, not so much by the radius of the main mass. In the present case the protrusion apparently had a mass of about $10^{5} \mathrm{kilograms}$ and a radius of 1.4 meters.

Is it a coincidence that the cosmogenic rare gases in Canyon Diablo 3 , specimen 586.1 can be explained with the same set of assumptions? It too could have come from a location in the main mass which was exposed for 1.0 aeon and survived the collision 540 million years ago. Or it could likewise have come from a protrusion of about $10^{5}$ kilograms which was exposed during 540 million years.

If all the varieties of the Canyon Diablo meteorite indeed come from a single parent mass, then it follows that substantial structural and compositional variations existed in the nickeliron phase of the parent body of Canyon Diablo over distances of 10-100 meters. This adds a new element of flexibility to all theories on the origin of iron meteorites. 
1. H. H. Nininger, Popular Astronomy 48, 328 (1940).

2. H. H. Nininger and A. D. Nininger, The Nininger Collection of Meteorites, Winslow, Arizona, p. 129 and Plate VIII, Fig. 9 (1950).

3. E. A. Goldberg, A. Uchiyama and H. Brown, Geochim. et Cosmochim. Acta 2 , 1 (1951).

4. H. Brown, J. Geophys. Res. 66, 1316 (1961).

5. I have assumed that $6.7 \%$ of all meteorite falls are irons.

6. M. Honda, J. P. Shedlovsky and J. R. Arnold, Geochim. et Cosmochim. Acta 22, 133 (1961).

7. E. Öpik, Irish Astron. Journ. 5, 14 (1958).

8. E. M. Shoemaker, Report of the International Geological Congress, XXI Session, Norden. Part XVIII, p. 418 (1960).

9. R. L. Bjork, J. Geophys. Res. 66, 3379 (1961).

10. P. Signer and A. O. Nier, J. Geophys. Res. 65, 2947 (1960).

11. O. A. Schaeffer and D. Heymann, to be published.

12. M. E. Lipschutz and D. Heymann, to be published.

13. H. Voshage and D. C. Hess, Z. f. Naturforschg. 17a, 341 (1964).

14. E. Anders, Rev. Mod. Physics 34, 287 (1962).

15. There is good evidence that iron meteorites suffer repeated collisions in space which produce new surfaces. Fragments from under such new surfaces have shorter exposure ages than the main mass (see: E. Vilcsek and H. Wanke, Proceedings of the Symposium on Radioactive Dating, Athens 1962, p. 381 . IAEA, Vienna 1633). I myself have measured four Canyon Diablo 1 specimens not shown in Fig. 1, which indicate another, still 



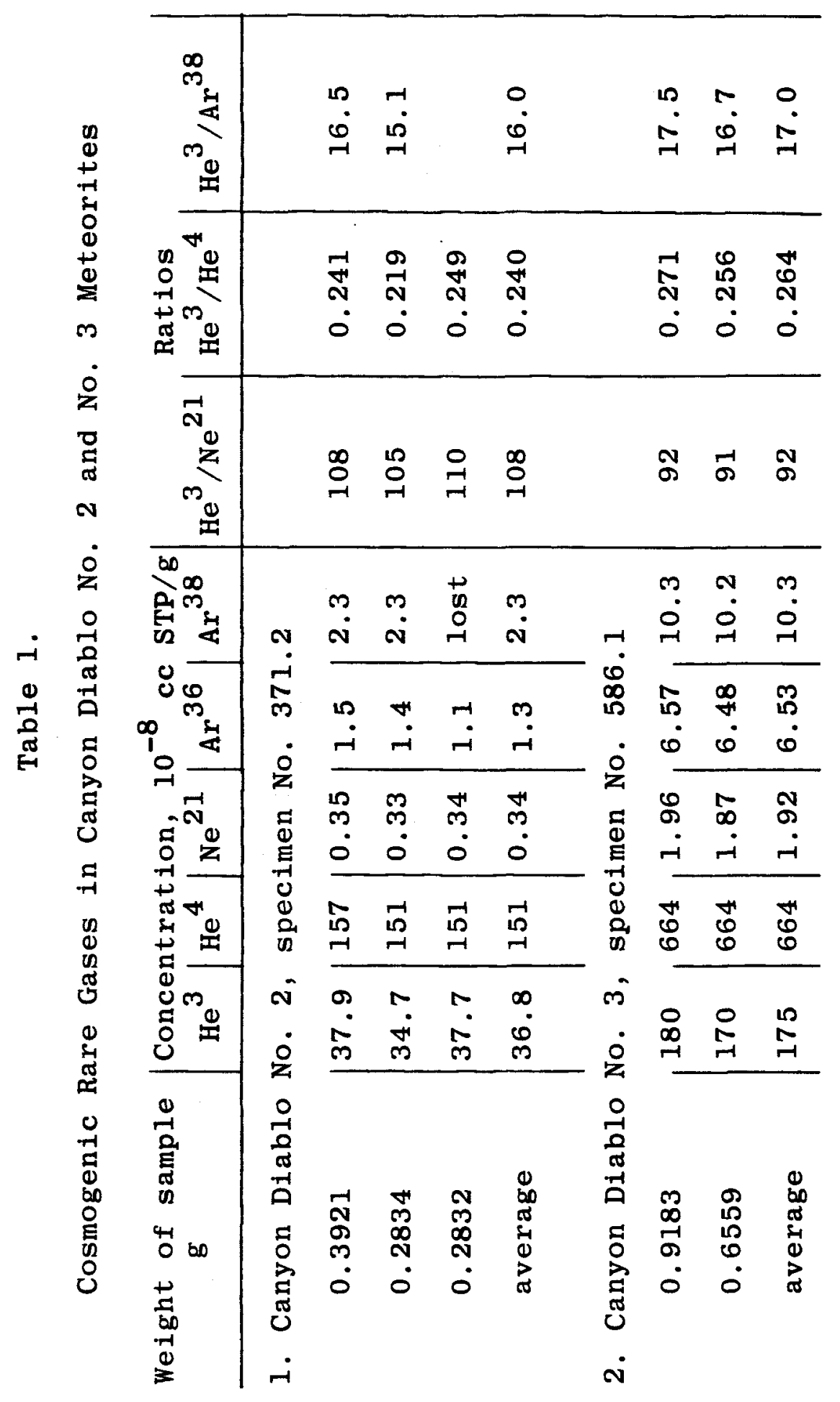




\section{Figure Caption}

- Fig. 1. $\mathrm{He}^{3} / \mathrm{Ne}^{21}$ vs. $\mathrm{Ar}^{38}$ for spherical meteoroids of increasing radius. The drawn curves are Signer and Nier's (10) calculated curves. Numbered points are measurements on Canyon Diablo 1. 


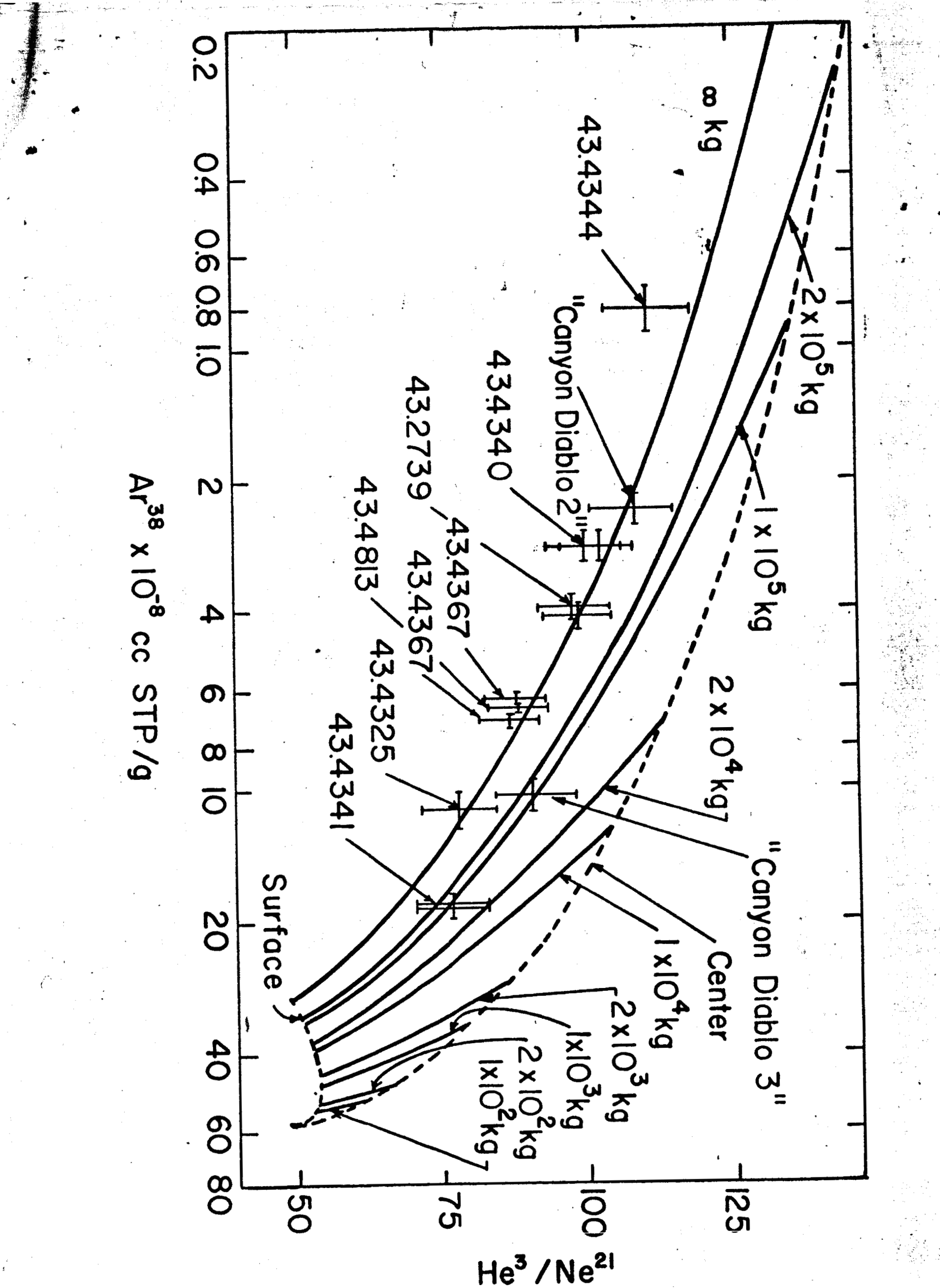

